\title{
PANCREAS
}

\section{Microglial activation key to pain in chronic pancreatitis}

New research has revealed that activation of the spinal microglia has a vital role in the initiation and maintenance of pain in a rat model of chronic pancreatitis, and provides insights into possible new drug targets when treating pain associated with pancreatic inflammation.

"In daily clinical practice, we often face chronic and intractable pain in patients with chronic pancreatitis, which is most often caused by excessive alcohol ingestion," says author Ching-Liang Lu (National Yang-Ming University and Taipei Veterans General Hospital, Taiwan). "Because of the unclear pathogenesis, the control of pain in patients with chronic pancreatitis remains difficult and frustrating," he adds.

As existing evidence indicated that the pain in chronic pancreatitis shared many characteristics of neuropathic pain, Lu and colleagues hypothesized that activation of the spinal microglia-important in the initiation and maintenance of chronic pain of neuropathic or inflammatory originmight also mediate the chronic visceral pain observed in patients with chronic pancreatic inflammation.

The researchers used a rat model of chronic pancreatitis that mimicked the behavioral and pathological changes in human disease. Chronic pancreatitis was induced by addition of trinitrobenzene sulfonic acid (TNBS) into the biliopancreatic ducts, and hyperalgesic responses were measured by monitoring the mechanical sensitivity of the abdomen (withdrawal response) and the nocifensive behavior (reflex responses to pain, including stretching and contraction of abdominal wall muscles) to electrical stimulation of the pancreas.

Lu and co-workers noted a shift in the status of the spinal microglia (in the dorsal horn of the thoracic spinal cord) from a resting to an activated state 3 weeks after inducing chronic pancreatitis in the rats; this shift in status was associated with an increased sensitivity to pain and an increase in nociceptive responses in the rats, and was thought to involve p38 MAPK pathway signaling. Interestingly, injection of minocycline (a microglial inhibitor) successfully treated, and also prevented, the pain responses in rats with TNBS-induced chronic pancreatitis.

The study authors now hope to explore the molecular mechanisms that mediate the crosstalk between the spinal microglia and neurons as well as the putative signaling pathways between the activated spinal microglia and the inflamed pancreas. "With this approach, we might provide chances for new drug development in the treatment of pain in patients with chronic pancreatitis by targeting the spinal microglia," notes Lu.

\section{Katrina Ray}

Original article Liu, P.-Y. et al. Spinal microglia initiate and maintain hyperalgesia in a rat model of chronic pancreatitis. Gastroenterology doi:10.1053/j.gastro.2011.09.041

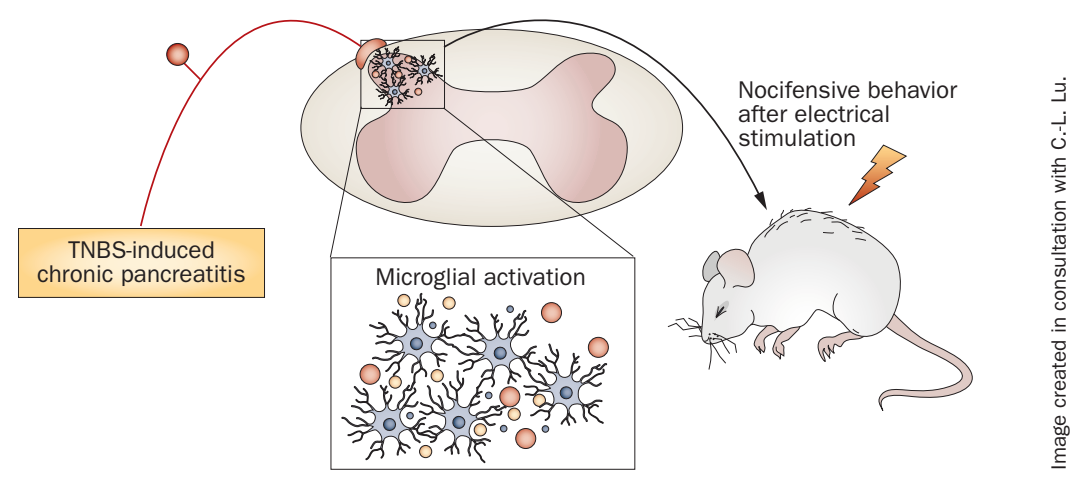

